\title{
Pneumococcus nasopharyngeal carriage in children attending an academic hospital in Pretoria, South Africa, after the introduction of pneumococcal vaccine
}

\section{Authors: \\ Charity Newton ${ }^{1}$ (I) \\ Harry Maake ${ }^{1}$ (D) \\ Caroline Maluleka ${ }^{2}$ (D) \\ Siyazi $\mathrm{Mda}^{3}$ (1) \\ Affiliations: \\ ${ }^{1}$ Department of Paediatrics and Child Health, Faculty of Health Sciences, Sefako Makgatho Health Sciences University, Pretoria, \\ South Africa}

${ }^{2}$ Department of Microbiology, Faculty of Health Sciences, Sefako Makgatho Health Sciences University, Pretoria, South Africa

${ }^{3}$ Faculty of Health Sciences, Nelson Mandela University, Port Elizabeth, South Africa

Corresponding author: Charity Newton, charitynewton@hotmail.com

Dates:

Received: 10 Jan. 2018 Accepted: 24 July 2019 Published: 22 Oct. 2019

How to cite this article: Newton C, Maake $\mathrm{H}$, Maluleka C, Mda S. Pneumococcus nasopharyngeal carriage in children attending an academic hospital in Pretoria, South Africa, after the introduction of pneumococcal vaccine. S Afr J Infect Dis. 2019;34(1), a112. https://doi. org/10.4102/sajid.v34i1.112

\section{Copyright:}

(C) 2019. The Authors. Licensee: AOSIS. This work is licensed under the Creative Commons Attribution License.

Read online:
Background: In 2009, pneumococcal conjugate vaccine was introduced in South Africa. However, there are concerns that this could lead to an increase in colonisation of non-vaccine serotypes (serotype replacement).

Methods: In a cross-sectional study, 350 children aged 1 month to 14 years were enrolled at Dr George Mukhari Academic Hospital from December 2015 to April 2016. We assessed the prevalence of nasopharyngeal colonisation with pneumococcus and characterised the serotypes found.

Results: The median age of the cohort was 33.7 months (interquartile range 16.27-69.5 months), with $20 \%$ being $<1$ year. A total of $21 \%$ of the children were diagnosed with pneumococcalrelated conditions; among these, pneumonia was the most common condition. Less than half $(43 \%)$ of the participants were fully immunised. Forty-six $(13 \%)$ of the children were colonised with pneumococcus. Younger age was significantly associated with pneumococcal colonisation. Among those colonised with pneumococcus, 35\% were fully immunised, 30\% were partially immunised, 30\% had an unknown immunisation status and $4 \%$ were unimmunised. Eight $(17 \%)$ of the children who were colonised with pneumococcus had pneumococcal-related conditions. The commonest serotype identified was 6A/B. Overall, $2 \%$ of the cohort were colonised with vaccine-serotype pneumococcus.

Conclusion: As a minority of children had evidence of nasopharyngeal colonisation with vaccine-serotype pneumococci, serotype replacement may be emerging in our population.

Keywords: Children; pneumococcal nasopharyngeal colonisation; immunisation; serotypes; serotype replacement.

\section{Introduction}

It is estimated that pneumococcal disease accounts for about 300000 deaths in children, and half of these occur in Africa. ${ }^{1}$ The incidence of pneumococcal disease differs in many populations and factors such as overcrowding, poverty, human immunodeficiency virus (HIV) and genetics have been attributed as contributing to these differences. ${ }^{2}$

Streptococcus pneumoniae (pneumococcus) is part of the commensal flora of the respiratory tract., Nasopharyngeal pneumococcal colonisation is common in children ${ }^{5}$ and it is a prerequisite for developing pneumococcal disease. ${ }^{2,4,6}$ Colonisation is the main source for transmission of the bacterium within communities; therefore, preventing nasopharyngeal colonisation in children is a strategy for preventing disease in children and adults. ${ }^{4}$ Immunisation of children with pneumococcal conjugate vaccine (PCV) has been shown to decrease the risk of acquiring vaccine serotype nasopharyngeal colonisation.?

There are more than 90 pneumococcal serotypes;, 89 however, before the introduction of PCV, about 10 serotypes accounted for more than $70 \%$ of invasive pneumococcal disease (IPD) in children globally. ${ }^{9}$ Invasive pneumococcal disease is defined as isolation of pneumococcus from a normally sterile site, for example, cerebrospinal fluid, blood, joint fluid and pleural fluid..$^{9,10,11}$ It includes pneumonia, septicaemia and meningitis caused by pneumococcus. ${ }^{9}$

In 2009, South Africa introduced PCV into its routine immunisation programme. ${ }^{12}$ The 7 -valent PCV (PCV7) that targets serotypes $4,6 \mathrm{~B}, 9 \mathrm{~V}, 14,18 \mathrm{C}, 19 \mathrm{~F}$ and $23 \mathrm{~F}^{11}$ was introduced with two 
primary doses, given to infants at 6 and 14 weeks of age and a booster at 9 months. ${ }^{13}$ In 2011, a 13-valent PCV (PCV13) replaced PCV7.14 In addition to serotypes contained in PCV7, PCV13 includes serotypes 1, 3, 5, 6A, 7F and 19A. ${ }^{11,15}$ Surveillance among South African children from 2005 to 2012 showed that the introduction of PCV7 had been effective in reducing the rate of PCV7 serotype IPD. ${ }^{13}$

Some studies in Europe and the United States after the introduction of PCV have shown an increase in the prevalence of carriage of serotypes not included in the vaccine (nonvaccine serotypes [NVTs]), a phenomenon called serotype replacement. ${ }^{16,17}$ Similarly, in South Africa there has been an increase in NVT colonisation, although there has been no significant increase in NVT IPD. ${ }^{18}$

It is essential to monitor the pneumococcal colonisation prevalence in South African children subsequent to PCV introduction, and to characterise the colonising serotypes. The current study was designed to assess nasopharyngeal colonisation prevalence in South African children subsequent to PCV introduction, and to characterise the serotypes.

\section{Methods}

In a cross-sectional descriptive study, children aged 1 month to 14 years attending the general outpatients department at Dr George Mukhari Academic Hospital in Pretoria, South Africa, were sequentially enrolled. Sample size calculations were performed using the prevalence formula $n=Z^{2} P(1-P) / d^{2}$, where $Z=1.96$ for confidence, $P=$ expected prevalence and $d=0.05 .{ }^{19}$ Prevalence rates of $17 \%$ and $67 \%$ have been reported among Kenyan and South African study populations, respectively; ${ }^{20,21}$ these yielded sample sizes of 217 and 340, respectively. It was decided to enrol 350 children.

Exclusion criteria for the study included the following: children presenting with severe respiratory distress were excluded from the study.

The following information was documented: date of birth (used to calculate age), sex and clinical diagnosis. Children with meningitis, pneumonia, otitis media or tonsillitis were designated as having pneumococcal-related conditions.

Participant anthropometry (weight, length and/or height) was measured by the principal investigator with the help of an assistant. Each child's weight was measured (approximated to the nearest $0.1 \mathrm{~kg}$ ), using a single calibrated scale, with the child only wearing a clean nappy or underwear. Length was measured using a measuring board with a fixed upper part and a moveable lower part as per validated procedures. ${ }^{22}$ Height was measured with the child standing upright against a stadiometer, according to standardised procedures. ${ }^{22}$ Length and height were approximated to the nearest $0.1 \mathrm{~cm}$. The age, weight and length or height were used to calculate weight-forage Z-scores, weight-for-height Z-scores (WHZ) and height-forage Z-scores (HAZ) using Epi-info software.
Participant HIV status was assigned by abstracting the result from clinical records. If an HIV result could not be obtained, the HIV status of the child was regarded as unknown.

The immunisation status of each child was obtained from their Road-to-Health card. A fully immunised child was described as a child who had received PCV doses at 6 weeks, 14 weeks and 9 months of age. A child who had not received all immunisations (including the child who was too young to have received all immunisations) was described as partially immunised and, if the child had not received any immunisations, the child was described as unimmunised. If the Road-to-Health card was not available, immunisation status was recorded as unknown.

Nasopharyngeal swabs were collected from all enrolled children by the principal investigator, according to World Health Organization-recommended standards ${ }^{23}$ and dispatched in Amies transport media for culture to the local National Health Laboratory Service laboratory within $3 \mathrm{~h}$ of collection. Swabs were inoculated onto 5\% sheep blood agar plates (Diagnostic Media Products, Johannesburg, South Africa) and incubated at $37^{\circ} \mathrm{C}$ for $18-24 \mathrm{~h}$.

Streptococcus pneumoniae was confirmed by appearance of colony morphology, gram stain, alpha haemolysis, optochin susceptibility and bile solubility testing. Streptococcus pneumoniae strain ATCC 49619 was used as a positive control, and the growth of Streptococcus mitis strain ATCC 49456 was used as a negative control.

Preserved pneumococcal isolates were used for DNA extraction using the ZR Genomic DNA-Tissue MiniPrep kit (Zymo Research Corporation, Irvine, CA, USA). Extracted DNA was stored at $-20^{\circ} \mathrm{C}$ and used as a template for polymerase chain reaction (PCR). The DNA extracted was quantified using a NanoDrop Lite Spectrophotometer (Thermo Scientific, Waltham, MA, US) with a purity of above 2 (A260/280). Primers were grouped into nine multiplex reactions, with four primer pairs targeting serotype-specific sequences of the four different serotypes in each reaction. We were able to detect the following vaccine serotypes: 1, 3, 4, 5, 6A/B, 7F, 9V, 14, 19A, 19F, 23F and 18C (included in sg18). The following NVTs were also detectable: 7C, 8F, 10A, 11A, 12F, 15A, 15B/C, 16F, 17F, 20, 22F, 31, 33F, 34, 35B, 35F and 38. Pneumococcal isolates that could not be typed using the primers stated in this study were designated as unknown serotypes.

\section{Statistical analysis}

Statistical analysis was performed using the IBM SPSS Statistics 23. Descriptive statistics including frequencies were utilised. Median with interquartile range (IQR) was used for age and anthropometric indices, as these variables were not normally distributed.

Anthropometric indices were compared between the two groups (those who tested positive for pneumococcal carriage 
and those who tested negative) using the Mann-Whitney $U$ test. The chi-square test was used to compare the frequency of HIV and immunisation status and other categorical variables between the two groups. Statistical significance was set at $p<0.05$ for all tests.

\section{Ethical consideration}

Ethical clearance was obtained from the Sefako Makgatho Health Sciences University Research Ethical Committee (SMUREC) prior to data collection (clearance number: SMUREC/M/47/2015/PG). Signed informed consent was obtained from the children's parents or caregivers.

\section{Results}

A total of 350 children were enrolled from December 2015 to April 2016, 44\% $(n=153)$ of whom were female. The median age was 33.7 months (IQR of 16.3-69.5 months). The majority of the children $(n=242,69.1 \%)$ were under 5 years of age.

The median length or height was $89 \mathrm{~cm}$ (IQR, $74.5 \mathrm{~cm}-$ $111 \mathrm{~cm}$ ) and the median weight was $12.7 \mathrm{~kg}$ (IQR: $9.4 \mathrm{~kg}-$ $18.3 \mathrm{~kg}$ ). The median HAZ was -0.97 (IQR: -2.08 to 0.0 ), while that for weight-for-age was -1.02 (IQR, -1.82 to -0.48 ). The median WHZ was -0.17 (IQR: -1.17 to 0.78 ).

On assessment of the immunisation status, 150 (43\%) were fully immunised, 48 (18\%) were partially immunised, $66(19 \%)$ were not immunised and $86(24 \%)$ had undetermined immunisation status. There were 256 children who were either 10 months and older or were born after December 2009 (i.e. after the introduction of PCV). The immunisation status of these 256 children is shown in Table 1.

In terms of diagnosis, $74(21 \%)$ of the children had pneumococcal-related conditions. Among these, pneumonia

TABLE 1: Immunisation status of children born after pneumococcal conjugate vaccine introduction and who were 10 months or older.

\begin{tabular}{lcc}
\hline Immunisation status & Frequency & Proportion (\%) \\
\hline Fully immunised & 148 & 58 \\
Partially immunised & 74 & 29 \\
Not immunised & 26 & 10 \\
No RTHC (unknown status) & 8 & 3 \\
\hline Total & $\mathbf{2 5 6}$ & $\mathbf{1 0 0}$ \\
\hline
\end{tabular}

RTHC, Road-to-Health card. was the most common condition, with $60(17 \%)$ children out of the 350 enrolled having this diagnosis. There were eight children with tonsillitis, five had meningitis and one had otitis media. The remaining 276 children had conditions not deemed to be pneumococcal-related. Among children with pneumococcal-related diagnoses, $42 \%(25 / 60)$ of those with pneumonia were fully immunised, as were $60 \%(3 / 5)$ of those with meningitis and $63 \%$ (5/8) of those with tonsillitis. The child with otitis media was fully immunised against pneumococcus.

Pneumococcus was isolated in nasopharyngeal swabs in 13\% $(n=46)$. Children who were pneumococcus-positive were significantly younger (24.5 months; IQR: 9.5-48.1 months) than those who were pneumococcus-negative (34.8 months; IQR: 17.4-71.8 months), Mann-Whitney $U$ test, $p=0.007$. Sixteen $(35 \%)$ of the 46 children with positive pneumococcal nasopharyngeal carriage were fully immunised, compared to $137(45 \%)$ of the 304 who were pneumococcal carriage negative. There was no association between nasopharyngeal pneumococcal carriage and immunisation status among children eligible to have received a full course of PCV immunisation $(p>0.1)$. Eight $(17 \%)$ of the children who were colonised with pneumococcus had pneumococcal-related conditions. Six of the eight had pneumonia and two had tonsillitis.

Anthropometric indices were similar in children who tested positive for pneumococcus colonisation and those who were pneumococcus-negative (Table 2).

With regard to the HIV status, 307 (88\%) were HIV-negative, 33 (9\%) were HIV-positive and 10 (3\%) had an unknown status. Forty $(87 \%)$ children positive for pneumococcus colonisation were HIV-negative. Of the 304 children who were negative for pneumococcus on nasopharyngeal testing, 267 (87.8\%) were HIV-negative, 27 (8.9\%) were HIV-positive and $10(3.3 \%)$ had an unknown HIV status (Table 3). There was no statistically significant association between HIV status and pneumococcal nasopharyngeal carriage $(p>0.1)$.

Polymerase chain reaction testing for pneumococcal serotypes was conducted on all 46 pneumococcal-positive cases, and nine $(20 \%)$ of them were positive for serotypes included in our PCR assays. Five serotypes were detected among these

TABLE 2: Age, gender, anthropometric indices and human immunodeficiency virus status of enrolled children in relation to their pneumococcal carriage.

\begin{tabular}{|c|c|c|c|c|c|c|c|c|c|c|}
\hline \multirow[t]{3}{*}{ Variable } & \multicolumn{10}{|c|}{ Pneumococcal carriage } \\
\hline & \multicolumn{4}{|c|}{ Positive $(n=46)$} & \multicolumn{3}{|c|}{ Negative $(n=304)$} & \multicolumn{3}{|c|}{ All children $(n=350)$} \\
\hline & Median & IQR & $n$ & $\%$ & Median & IQR & $\%$ & Median & IQR & $\%$ \\
\hline Age in months & 24.5 & $9.5-48.1^{*}$ & & - & 34.8 & $17.4-71.8$ & - & 33.7 & $16.3-69.5$ & - \\
\hline Females & - & - & 24 & 52.1 & $129 / 304$ & - & 42.4 & $153 / 350$ & - & 43.7 \\
\hline WAZ & -1.45 & -2.20 to -0.20 & - & - & -0.93 & -1.80 to -0.004 & - & -1.02 & -1.82 to +0.48 & - \\
\hline HAZ & -1.01 & -2.16 to -0.02 & - & - & -0.97 & -2.07 to 0.00 & - & -0.97 & -2.08 to 0.00 & - \\
\hline HIV-positive & - & - & 6 & 13.0 & $27 / 304$ & - & 8.9 & $33 / 350$ & - & 9.4 \\
\hline
\end{tabular}

WAZ, weight-for-age Z-score; WHZ, weight-for-height Z-score; HAZ, height-for-age Z-score; HIV, human immunodeficiency virus; IQR, interquartile range.

Note: Values for age, WAZ, HAZ and WAZ stated as median (interquartile range), except for gender and HIV-positive, which are stated as $n(\%)$.

*, Significantly lower than pneumococcal negative group, $p<0.05$. 
TABLE 3: Pneumococcal carriage results of study children by their human immunodeficiency virus status.

\begin{tabular}{|c|c|c|c|c|c|c|}
\hline \multirow{2}{*}{ Pneumococcus } & \multicolumn{2}{|c|}{ HIV-positive } & \multicolumn{2}{|c|}{ HIV-negative } & \multicolumn{2}{|c|}{ HIV unknown } \\
\hline & $n$ & $\%$ & $n$ & $\%$ & $n$ & $\%$ \\
\hline Positive $(n=46)$ & 6 & 13.0 & 40 & 87.0 & 0 & 0.0 \\
\hline Negative $(n=304)$ & 267 & 87.8 & 27 & 8.9 & 10 & 3.3 \\
\hline
\end{tabular}

HIV, human immunodeficiency virus.

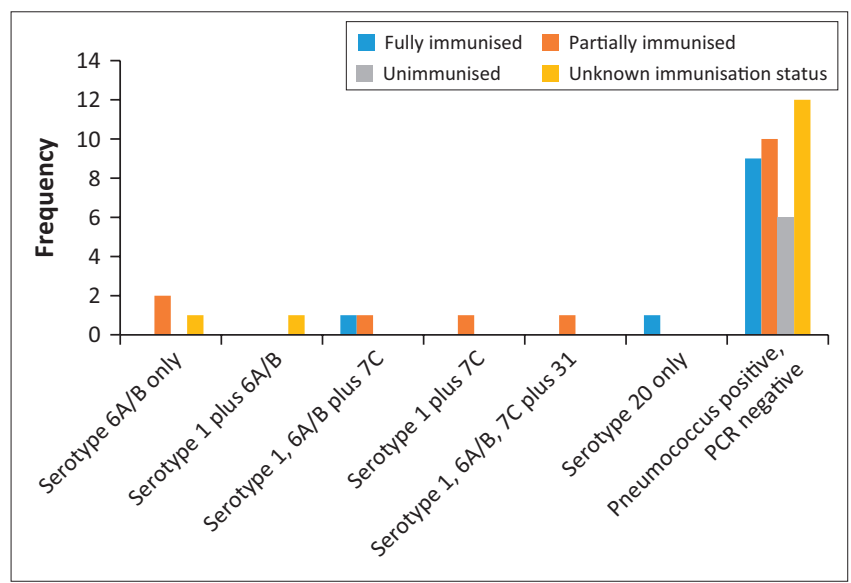

FIGURE 1: Pneumococcal serotypes identified among 46 children who had pneumococcal nasopharyngeal carriage.

nine children: two vaccine serotypes ( 1 and $6 \mathrm{~A} / \mathrm{B})$ and three NVTs (7C, 20 and 31). Four children had carriage of a single serotype: three with $6 \mathrm{~A} / \mathrm{B}$ and one with serotype 20 . Five children had carriage of more than one serotype: one child had serotypes 1 and $6 \mathrm{~A} / \mathrm{B}$, one child had serotypes 1 and $7 \mathrm{C}$, two had serotypes $1,6 \mathrm{~A} / \mathrm{B}$ and $7 \mathrm{C}$ and one had carriage of serotypes 1, 6A/B, 7C and 31. Serotype 6A/B was detected in seven $(77.8 \%)$ of the nine children positive for pneumococcus on PCR testing. Serotype 7C was the commonest NVT identified (detected in four [44.4\%] of the nine children). Figure 1 illustrates the pneumococcal serotypes among the 46 children whose nasopharyngeal swabs cultured pneumococcus. In the eight children with pneumococcalrelated diagnoses and in whom nasopharyngeal swabs cultured pneumococcus, four were PCR-negative for the serotypes included in our assays. Of the four children with PCR-positive results, one had serotype $6 \mathrm{~A} / \mathrm{B}$, one had serotypes 1 and $7 \mathrm{C}$, one had serotypes $1,6 \mathrm{~A} / \mathrm{B}$ and $7 \mathrm{C}$ and one had serotypes $1,6 \mathrm{~A} / \mathrm{B}, 7 \mathrm{C}$ and 31 .

Overall, $8(2 \%)$ of the 350 children were colonised with vaccine serotypes, $5(1 \%)$ carried NVTs and $37(11 \%)$ carried pneumococci that could not be serotyped by the assays which we used. Of the eight children colonised with vaccineserotype pneumococci, one was fully immunised, five were partially immunised and two had undetermined vaccination status.

Of the 256 children who were eligible to have been immunised with PCV, $32(13 \%)$ tested positive for pneumococcus. Of the 150 fully immunised children, 143 (95\%) were HIV-negative, 5 (3\%) were HIV-positive and $2(1 \%)$ had undetermined HIV status. There was no association between HIV and immunisation status $(p>0.1)$.
TABLE 4: Immunisation status and pneumococcal carriage of enrolled children.

\begin{tabular}{|c|c|c|c|c|c|c|}
\hline \multirow[t]{3}{*}{ Immunisations } & \multicolumn{4}{|c|}{ Pneumococcal carriage } & \multicolumn{2}{|c|}{ Total } \\
\hline & \multicolumn{2}{|c|}{ Positive } & \multicolumn{2}{|c|}{ Negative } & \multirow[b]{2}{*}{$n$} & \multirow[b]{2}{*}{$\%$} \\
\hline & $n$ & $\%$ & $n$ & $\%$ & & \\
\hline Fully immunised & 16 & 34.8 & 137 & 45.1 & 153 & 43.7 \\
\hline Partially immunised & 14 & 30.4 & 31 & 10.2 & 45 & 12.9 \\
\hline Not immunised & 2 & 4.3 & 64 & 21.1 & 66 & 18.9 \\
\hline No RTHC (unknown status) & 14 & 30.4 & 72 & 23.4 & 86 & 24.6 \\
\hline Total & 46 & 100 & 304 & 100 & 350 & 100 \\
\hline
\end{tabular}

RTHC, Road-to-Health card.

There was no statistically significant association between immunisation status and nasopharyngeal pneumococcal carriage (Table 4).

\section{Discussion}

In this study, we assessed the nasopharyngeal pneumococcal carriage prevalence in children attending an academic hospital in Pretoria approximately 7 years after the introduction of $\mathrm{PCV}$, and we documented a carriage rate of $13 \%$. This rate is lower than that noted in a South African trial conducted prior to the introduction of $\mathrm{PCV}$, where the carriage rate was found to be $54 \%$ among immunised children. ${ }^{24}$ In the Gambia, a carriage rate of $35 \%$ was noted, ${ }^{25}$ while in Kenya the carriage rate was $17 \%{ }^{20}$ The rate which we observed is similar to that noted in the Kenyan study. This rate of $13 \%$ is, however, considerably lower than the carriage rate of $47 \%$ which was observed in a South African study conducted 2 years after the roll-out of PCV. ${ }^{7}$

Vaccine serotype colonisation was found to be $2 \%$ in our study, which is lower than the $22 \%$ (in children 12 years and younger) found in a South African study conducted 2 years after the PCV roll-out; ${ }^{7}$ this is likely an effect of immunisation. It is possible that the decline in vaccine-type colonisation has continued; however, it should be noted that Amies transport medium was used in our study rather than skim milktryptone-glucose-glycerine (STGG), which is the preferred transport medium for pneumococcus. ${ }^{23}$ It is possible that using STGG may have improved the viability of the organism and thus resulted in a different colonisation rate.

Pneumococcus nasopharyngeal carriage was more likely to be present in younger children in our study, which is in line with findings from other studies. A South African study assessing pneumococcal colonisation noted a prevalence of $73 \%$ in children younger than 5 years and $50 \%$ in the 6-12 year age group. ${ }^{7}$ In the Gambia, pneumococcal carriage decreased with increasing age, with the highest prevalence observed in younger children. ${ }^{25} \mathrm{~A}$ systematic review on pneumococcal carriage in sub-Saharan Africa also confirmed that pneumococcal carriage was highest for children less than 5 years and decreased with age. ${ }^{26}$ On the other hand, in an Italian study, pneumococcal colonisation was found to rise with increasing age. ${ }^{27}$

The HIV prevalence in our cohort was $9.4 \%$, which is higher than the estimated vertical transmission rate of HIV from mother-to-child $(<5 \%)$ in South Africa. ${ }^{28}$ Similarly, another 
study had also shown a low mother-to-child transmission (MTCT) rate of $2.7 \% .^{29}$ However, in our study, the high prevalence rate was probably related to the fact that the children were enrolled from a hospital setting rather than from the general population. There was no correlation between HIV status and pneumococcal carriage. This was also corroborated in a study conducted in South Africa where no difference was found in carriage of pneumococcus in HIV-uninfected and HIV-infected children. ${ }^{30}$ In another South African study, however, the prevalence of pneumococcal colonisation in HIV-infected children was higher than those who were HIV-uninfected. ${ }^{31}$

In this study, only $43 \%$ of all enrolled children were fully immunised against pneumococcus. It is, however, important to note that some of the children were younger than 9 months; however, even among children who were 10 months or older and who were born after the introduction of PCV, 58\% were fully immunised. This immunisation coverage is much lower than the government target of $95 \%$ coverage for immunisations by 1 year. ${ }^{32}$ It was also lower than the suboptimal coverage found in previous South African studies, where one study recorded $75 \%$ immunisation coverage at 9 months when all PCV vaccinations are completed and another study recorded an immunisation coverage of $72 \%$ at 9 months. ${ }^{33,34}$ In one of the studies, vaccine stock-outs were mentioned as a major contributor to the low immunisation coverage. ${ }^{32}$ However, as our study was conducted in children who were in a hospital, the children were likely to be under-vaccinated, as a lack of vaccination is a risk for hospitalisation with pneumococcalrelated conditions.

In our study, no correlation was found between pneumococcal carriage and immunisation as the prevalence of pneumococcal colonisation was similar between children who were fully immunised and those who were partially immunised or not immunised. This has also been shown in other studies. ${ }^{27}$ In the Gambia, it was found that the overall prevalence of pneumococci was similar in fully immunised and partially immunised villages, with a trend towards lower prevalence among children (5-15 years age group) in fully immunised communities. ${ }^{25}$

In our study, the most common vaccine serotype was 6A/B, followed by serotype 1 . Serotypes $6 \mathrm{~A}$ and $6 \mathrm{~B}$ could not be separated due to a lack of equipment to do pyrosequencing. Nonetheless, most pneumococcal isolates (80\%) were negative for the serotypes included in our PCR assays. It should be noted that we tested for approximately onethird of the known serotypes, with adequate positive and negative controls built into the assay. We, however, only performed PCR testing on pneumococcal culture-positive nasopharyngeal swabs. Similarly, we could not test for all NVTs because of financial limitations.

Serotype 6A/B is commonly reported in studies assessing nasopharyngeal colonisation, including surveys from South Africa, Kenya, and a meta-analysis of studies conducted in low and lower middle-income countries. ${ }^{7,20,35}$ None of the samples were positive for serotype $15 \mathrm{~B}$, which was the commonest NVT causing IPD prior to the introduction of PCV. ${ }^{36}$ Nonetheless, the majority of pneumococcal isolates were not serotyped by our PCR assays. These were probably NVTs that were not included in the assays. The preponderance of these isolates is suggestive of serotype replacement.

We consider our inability to define the serotypes of the majority of the pneumococcal isolates obtained in our study, together with the use of Amies transport medium (rather than STGG) to be important limitations of the study.

The introduction of PCV and maintenance of high levels of vaccine coverage are expected to lead to a decrease in vaccine serotype colonisation and possibly achieve herd protection. While our study suggested a lower than previously reported prevalence of pneumococcal nasopharyngeal carriage in South African children, the stated limitations may have confounded the results.

Our findings indicate that serotype replacement colonisation is likely to have taken place. However, this needs to be confirmed in subsequent studies that are designed to enhance the yield and serotyping of pneumococcus.

\section{Acknowledgement}

The authors would like to extend their appreciation to the parents who gave consent for their children to be part of the study and the laboratory personnel who worked on the samples.

\section{Competing interests}

The authors have declared that no competing interests exists.

\section{Authors' contributions}

All the authors contributed equally to this work.

\section{Funding information}

This research received no specific grant from any funding agency in the public, commercial or not-for-profit sectors.

\section{Data availability statement}

Data sharing is not applicable to this article as no new data were created or analysed in this study.

\section{Disclaimer}

The views and opinions expressed in this article are those of the authors and do not necessarily reflect the official policy or position of any affiliated agency of the authors.

\section{References}

1. Wahl B, O'Brian KL, Greenbaum A, et al. Burden of Streptococcus pneumoniae and Haemophilus influenza type B disease in children in the era of conjugate vaccines: Global, regional, and national estimates for 2000-15. Lancet Glob Health. 2018;6(7):e744-e757. https://doi.org/10.1016/S2214-109X(18)30247-X 
2. Cohen R, Levy C. 13-valent pneumococcal conjugate vaccine in Africa. Lancet Glob Health. 2017;5(3):e244-e245. https://doi.org/10.1016/S2214-109X(17)30044-X

3. Donkor ES, Annan JA, Badoe EV, Dayie NTKD, Labi A, Slotved H. Pneumococcal carriage among HIV infected children in Accra, Ghana. BMC Infect Dis. 2017;17(1):133. https://doi.org/10.1186/s12879-017-2224-0

4. Nunes MC, Jones SA, Groome MJ, et al. Acquisition of Streptococcus pneumoniae in South African children vaccinated with 7-varent pneumococcal conjugate vaccine at 6, 14 and 40 weeks of age. Vaccine. 2014;33(5):628-634. https://doi. org/10.1016/j.vaccine.2014.12.023

5. Mehtälä J, Antonio M, Kaltoft MS, O'Brien KL, Auranen K. Competition between Streptococcus pneumoniae strains: Implications for vaccine-induced replacement in colonization and disease. Epidemiology. 2013;24(4):522-529. https://doi. org/10.1097/EDE.0b013e318294be89

6. Madhi SA, Cohen C, Von Gottberg A. Introduction of pneumococcal conjugate vaccine into the public immunization program in South Africa: Translating research into policy. Vaccine. 2012;30(3)S:C21-C27. https://doi.org/10.1016/j.vaccine. 2012.05.055

7. Nzenze SA, Shiri T, Nunes MC. Temporal changes in pneumococcal colonization in a rural African community with high HIV prevalence following routine infant pneumococcal immunization. Pediatr Infect Dis J. 2013;32(11):1270-1278. https://doi.org/10.1097/01.inf.0000435805.25366.64

8. Dube FS, Ramjith J, Gardner-Lubbe S, et al. Longitudinal characterization of nasopharyngeal colonization with Streptococccal pneumoniae in a South African birth cohort post 13 -valent pneumococcal conjugate vaccine implementation. Sc Rep. 2018;8(1):12497.

9. World Health Organization (WHO). Pneumococcal vaccines WHO position paper 2012. Weekly epidemiological record. 2012;87:129-144.

10. Von Mollendorf C, Von Gottberg A, Tempia S, et al. Increased risk for and mortality from invasive pneumococcal disease in HIV-exposed but uninfected infants aged $<1$ year in South Africa, 2009-2013. Clin Infect Dis. 2015;60(9):1346-1356. $<1$ year in South Africa, 2009-2013
https://doi.org/10.1093/cid/civ059

11. Waight PA, Andrews JN, Ladhani SN, Sheppard CL, Slack MPE, Miller E. Effect of the 13-valent pneumococcal conjugate vaccine on invasive pneumococcal disease in England and Wales 4 years after its introduction: An observational cohort study. Lancet Infect Dis. 2015;15(5):535-543. https://doi.org/10.1016/S1473-3099(15) Lancet In

12. Madhi SA, Bamford L, Ngcobo N. Effectiveness of pneumococcal conjugate vaccine and rotavirus vaccine introduction into the South African public immunization programme. S Afric Med J. 2014;104(4):228-234. http://www.samj.org.za/index. php/samj/article/view/7597.

13. Von Gottberg A, De Gouveia L, Tempia S. Effects of vaccination on invasive pneumococcal disease in South Africa. N Engl J Med. 2014;371(20):1889-1899. pneumococcal disease in South Africa. N
https://doi.org/10.1056/NEJMoa1401914

14. Cohen C, Von Mollendorf C, De Gouveia L, et al. Effectiveness of the 13-valent pneumococcal conjugate vaccine against invasive pneumococcal disease in South African children: A case-control study. Lancet Glob Health. 2017;5(3):e359-e369. https://doi.org/10.1016/S2214-109X(17)30043-8

15. Von Gottberg A, Cohen C, De Gouveia L, et al., 2003-2008. Epidemiology of invasive pneumococcal disease in the pre-conjugate vaccine era: South Africa. Vaccine. 2013;31(38):4200-4208. https://doi.org/10.1016/j.vaccine.2013.04.077

16. Hanquet $G$, Kissling $E$, Fenoll $A$, et al. Pneumococcal serotypes in children in 4 European countries. Emerg Infect Dis. 2010;16(9):1428-1439. https://doi.org/ 10.3201/eid1609.100102

17. Huang SS, Hinrichsen VL, Stevenson AE, et al. Continued impact of pneumococcal conjugate vaccine on carriage in young children. Pediatrics. 2009;124(1):e1-e11. $\mathrm{https}: / /$ doi.org/10.1542/peds.2008-3099

18. Nzenze SA, Madhi SA, Shiri T, et al. Imputing the direct and indirect effectiveness of childhood pneumococcal conjugate vaccine against invasive pneumococcal disease by surveying temporal changes in nasopharyngeal pneumococcal colonization. Am J Epidemiol. 2017;186(4):435-444. https://doi.org/10.1542/ peds.2008-3099

19. Naing L, Winn T, Rusli BN. Practical issues in calculating the sample size for prevalence studies. Arch Orofacial Sci. 2006;1:9-14.
20. Githii S, Revathi G, Muigai A, Kariuki S. Carriage rate and serotypes of Streptococcus pneumoniae amongst children in Thika Hospital, Kenya. Afr J Lab Med. 2013;2(1):5. https://doi.org/10.4102/ajlm.v2i1.45

21. Nzenze SA, Shiri T, Nunes MC, et al. Temporal association of infant immunisation with pneumococcal conjugate vaccine on the ecology of Streptococcus pneumoniae, Haemophilus influenzae and Staphylococcus aureus nasopharyngeal colonisation in a rural South African community. Vaccine. 2014;32(42):5520-5530. https://doi. org/10.1016/j.vaccine.2014.06.091

22. The United Nations Children's Fund (UNICEF). The state of the world's children 2012. New York: UNICEF; 2012.

23. Satzke C, Turner P, Virolainen-Julkunen A, et al. Standard method for detecting upper respiratory carriage of Streptococcus pneumoniae: Updated recommendation from the World Health Organization Pneumococcal Carriage Working Group. Vaccine. 2013;32(1):165-179. https://doi.org/10.1016/j.vaccine.2013.08.062

24. Mbelle N, Huebner RE, Wasas AD, Kimura A, Chang I, Klugman KP. Immunogenicity and impact on nasopharyngeal carriage of a nonavalent pneumococcal conjugate vaccine. J Infect Dis. 1999;180(4):1171-1176. https://doi.org/10.1086/315009

25. Roca A, Dione MM, Bojang A, et al. Nasopharyngeal carriage of pneumococci four years after community-wide vaccination with PCV-7 in the Gambia: Long-term evaluation of a cluster randomized trial. PLoS One. 2013;8(9):e72198. https://doi. org/10.1371/journal.pone.0072198

26. Usuf E, Bottomley C, Adegbola RA, Hall A. Pneumococcal carriage in sub-Saharan Africa: A systematic review. PLoS One. 2014;9(1):e85001. https://doi.org/10.1371/ journal.pone.0085001

27. Pasinato A, Indolfi $G$, Marchisio $P$, et al. Pneumococcal serotype distribution in 1315 nasopharyngeal swabs from a highly vaccinated cohort of Italian children as detected by RT-PCR. Vaccine. 2014;32(12):1375-1381. https://doi.org/10.1016/j. vaccine.2014.01.023

28. Goga AE, Dinh T, Jackson DJ, et al. Population-level effectiveness of PMTCT Option A on early mother-to-child (MTCT) transmission of HIV in South Africa: Implications for eliminating MTCT. J Glob Health. 2016;6(2):020405. https://doi. org/10.7189/jogh.06.020405

29. Bhardwaj S, Barron P, Pillay $Y$, et al. Elimination of mother-to-child transmission of HIV in South Africa: Rapid scale-up using quality improvement. S Afr Med J. 2014;104(3):239-243. https://doi.org/10.7196/SAMJ.7605

30. Nzenze SA, Von Gottberg A, Shiri T, et al. Temporal changes in pneumococcal colonization in HIV-infected and HIV-uninfected mother-child pairs following transitioning from 7-valent to 13-valent pneumococcal conjugate vaccine, Soweto, South Africa. J Infect Dis. 2015;212(7):1082-1092. https://doi.org/ 10.1093/infdis/jiv167

31. Ditse Z, Adrian PV, Kuwanda L, Madhi SA. Association of Streptococcus pneumoniae common protein antigen (CPA) antibodies and pneumococcal nasopharyngeal colonization in HIV-infected and HIV-uninfected African children. Vaccine. 2013;31(40):4421-4427. https://doi.org/10.1016/j.vaccine.2013.06.097

32. Le Roux K, Akin-Olugbade $O$, Katzen LS, et al. Immunisation coverage in the rural Eastern Cape - Are we getting the basics of primary care right? Results from a longitudinal prospective cohort study. S Afr Med J. 2017;107(1):52-55. https:// doi.org/10.7196/SAMJ.2017.v107i1.11242

33. Le Roux DM, Myer L, Nicol MP, Zar HJ. Incidence and severity of childhood pneumonia in the first year of life in a South African birth cohort: The Drakenstein Child Health Study. Lancet Glob Health. 2015;3(2):e95-e103. https://doi.org/ 10.1016/S2214-109X(14)70360-2

34. Zar HJ, Barnett W, Stadler A, Gardner-Lubbe S, Myer L, Nicol MP. Aetiology of childhood pneumonia in a well vaccinated South African birth cohort: A nested case-control study of the Drakenstein Child Health Study. Lancet Respir Med. 2016;4(6):463-472. https://doi.org/10.1016/S2213-2600(16)00096-5

35. Adegbola RA, DeAntonio R, Hill PC, et al. Carriage of Streptococcus pneumoniae and other respiratory bacterial pathogens in low and lower-middle income countries: A systematic review and meta-analysis. PLoS One. 2014;9(8):e103293. https://doi.org/10.1371/journal.pone.0103293

36. Ndlangisa KM, Du Plessis M, Wolter N, et al. Population snapshot of Streptococcus pneumoniae causing invasive disease in South Africa prior to introduction of pneumococcal conjugate vaccines. PLoS One. 2014;9(9):e107666. https://doi.org/ 10.1371/journal.pone.0107666 